\title{
Artificial Intelligence in Endoscopy
}

\author{
Helmut Messmann \\ III. Medizinische Klinik, Universitätsklinikum Augsburg \\ helmut.messmann@uk-augsburg.de
}

Artificial intelligence (AI) will revolutionize our daily life and will have tremendous impact on health care. Especially the influence in disciplines where imaging plays an important role seems to be substantial. Radiology, pathology and endoscopy will benefit from these developments. So far diagnosis of diseases by using images is based on the experience of the physician (radiologist, pathologist, endoscopist) and highly subjective with low inter- and intra-observer agreement.

Meanwhile AI has become routine in some parts of endoscopy such as screening colonoscopy. The quality of screening endoscopy depends on the number of detected polyps, which is called adenoma detection rate (ADR). Usually an ADR of at least $20 \%$ (women) or $25 \%$ (men) is recommended. Different techniques such as (virtual) chromo-endoscopy, caps on the distal end of the endoscope or optimizing withdrawal time have shown to increase ADR. By using AI first randomized trials could show a significant increase of ADR, mainly for small polyps $(<5 \mathrm{~mm})$. However, it is questionable whether these small polyps have any clinical impact. Besides detection the differentiation of polyps is of major impact. First prototypes showed the possibility to differentiate adenoma from non-adenoma polyps which is of clinical relevance.

Meanwhile similar efforts are made for gastric cancer or esophageal cancer. Our group was the first worldwide to show that AI can differentiate normal Barrett mucosa from dysplastic mucosa, which is a precursor of cancer. Meanwhile we are able to detect cancer real time during endoscopy.

Besides detection and differentiation of polyps and cancer the invasion depth of a cancer is of clinical importance. Usually endoscopic ultrasound is used for staging early cancers to predict whether endoscopic treatment or surgery is necessary. AI seems to have the potential to diagnose the invasion depth of early tumors and so guiding the optimal therapy.

In addition AI can control the endoscopist during his procedure to avoid incomplete visual observation of the Gi-tract. 